\title{
Outstanding Young Investigator Award Given to Alivisatos for Nanocrystal Research
}

A. Paul Alivisatos is the 1995 recipient of the Materials Research Society's Outstanding Young Investigator Award. Alivisatos, a professor in the Department of Chemistry at the University of California-Berkeley, is cited "for leadership in materials research, notably in the field of nanocrystals."

The Outstanding Young Investigator Award recognizes exceptional, interdisciplinary scientific work in materials research by a young scientist or engineer who also shows excellent promise as a developing leader in the materials area.

Alivisatos has gained recognition for his study of synthesis and characterization of semiconductor nanocrystals, which are clusters of $10 \mathrm{~s}$ to 10,000 s of atoms with electrical properties intermediate between those of bulk and molecular materials. For instance, they may have the crystalline order of bulk materials, but show bandgap changes brought about by their small size. The unique properties of these clusters can be retained after assembly of the clusters into defined structures, as Alivisatos has demonstrated.

His most significant scientific contributions relate to phase transitions in nanocrystals, nanocrystalline surface characterization, optical properties of nanocrystals, and incorporation of nanocrystals into light emitting diodes.

Alivisatos received a Bachelor's degree in chemistry in 1981 from the University of Chicago. He attended the University of California-Berkeley, where he earned his PhD degree with Charles Harris, professor of chemistry, based on the photophysics of electronically excited molecules near metal and semiconductor surfaces.

In 1986 he joined AT\&T Bell Laboratories in a postdoctoral position, where he was instrumental in setting up a collaborative research effort with Louis Brus and Michael Steigerwald. From this work new synthetic techniques involving arrested precipitation were developed to allow the production of CdS and CdSe nanocrystals with a narrow size distribution, within a variance of only a few percent.

In 1988, he joined the faculty of the University of California-Berkeley as an assistant professor of chemistry and was promoted to associate professor in 1992.

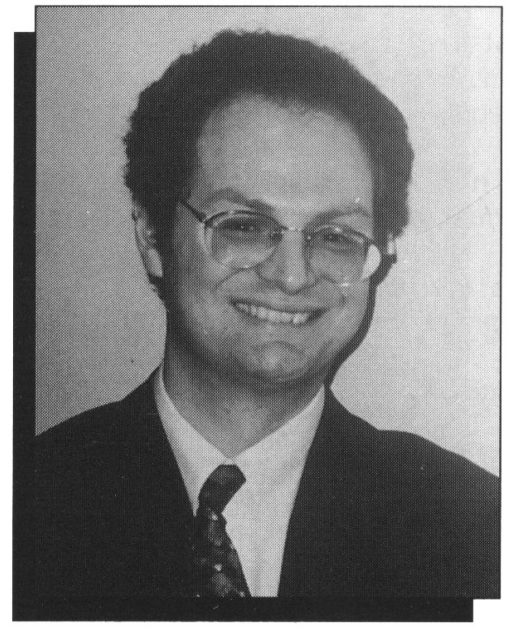

A. Paul Alivisatos

Last year he obtained the position of full professor.

Alivisatos has performed elegant studies of phase transitions in systems of finite size, and has constructed "phase diagrams" for these systems. In particular, he has measured the melting points of CdS particles as a function of their size and has determined the pressure dependence of solid-solid phase transitions in this system. By comparing his results to corresponding properties of bulk CdS, he was able to do the first systematic thermodynamic characterization of matter in this size regime.

What sets Alivisatos apart from his peers is his interdisciplinary and multifaceted approach to studying these nanocrystalline systems. His work requires a thorough understanding of the chemistry and physics of nanocrystals and he has mastered and applied an impressive array of spectroscopies to look at the surface composition of nanocrystals and to examine their optical and physical properties.

He has developed a new method for studying photoemission in nanocrystals. CdS particles are attached to a gold substrate by means of a dithiol alkane chain. The particles are ionized by synchrotron radiation, allowing one to map out their electronic energy levels via photoelectron spectroscopy. He also showed that in
CdSe nanocrystals, the surface ligand (trin-octylphosphine oxide) binds selectively to the $\mathrm{Cd}$ atoms, leaving the Se atoms exposed and vulnerable to oxidation.

Alivisatos pioneered the use for holeburning spectroscopy to obtain the electronic spectrum of size-selected nanocrystals. His work has aimed to understand electron-phonon coupling and the role of the surface in the electron-hole dynamics following electronic excitation. Through time-resolved experiments, performed in collaboration with Charles Shank, Alivisatos was able to distinguish these two effects in the dephasing of the excited state. He determined that the dipole moment of the excited state is large and very size-dependent, indicating a large electron-hole separation and a nonspherical charge distribution.

And, toward practical applications, Alivisatos has incorporated the nanocrystals into light-emitting diodes in which nanocrystals are the active element. Light emission arises from the recombination of holes injected into a layer of semiconducting $p$-paraphenylene vinylene (PPV) with electrons injected into a multilayer film of CdSe nanocrystals. The observed electroluminescence results from electron-hole recombination in the nanocrystals.

The color produced by the device, Alivisatos showed, can be controlled either by altering the size of the clusters (ranging from red to yellow) or by increasing the applied voltage to the device, which creates green emission from the polymer layer. This work has been described in his August 4, 1994 article in Nature on light-emitting diodes (with V.L. Colvin and M.C. Schlamp).

Among awards Alivisatos has received are a National Science Foundation Presidential Young Investigator Award, an Alfred P. Sloan Foundation Fellowship, the ACS Exxon Solid State Chemistry Fellowship, and the Coblentz Award. He is a member of the American Chemical Society, the American Physical Society, the American Association for the Advancement of Science, and the Materials Research Society.

The OYI award will be presented to Alivisatos on Monday evening, April 17 at the 1995 MRS Spring Meeting in San Francisco. He also will give a presentation, "Symmetry in the Structure and Spectroscopy of CdSe Nanocrystals," at 5 pm on Wednesday, April 19, in Symposium E. 